\title{
NECROSE ASSÉPTICA BILATERAL DE CABEÇA DE FÊMUR EM CADELA SEM RAÇA DEFINIDA - RELATO DE CASO
}

\author{
BRASIL, Fabricio Bernardo de Jesus ${ }^{1}$ \\ DANEZE, Edmilson Rodrigo ${ }^{2}$ \\ ZERBINATTI, Paulo Dernovbsek ${ }^{3}$ \\ OLIVEIRA, Maria Rosa Silva Barbosa Lacerda de ${ }^{3}$
}

\begin{abstract}
RESUMO: A Necrose Asséptica da Cabeça do Fêmur (NACF) é uma doença incapacitante que provoca diversas limitações nas atividades locomotoras do animal acometido. A maior incidência de inicio dos sinais da afecção é entre 6 e 7 meses de idade, com uma variação de 3 a 13 meses. Nesse contexto, o presente trabalho objetiva relatar um caso de NACF bilateral em uma cadela sem raça definida de aproximadamente cinco anos de idade e pesando 7 $\mathrm{kg}$, com histórico de incoordenação motora e instabilidade de membros posteriores. Como a mesma era jovem, optou-se pelo procedimento cirúrgico de ostectomia bilateral concomitante de cabeça e colo femorais como tratamento para a afecção. Decorridos 10 dias do procedimento, observou-se que o animal apresentava apoio leve dos membros; os pontos foram removidos e não foi encontrada nenhuma alteração relevante no local da incisão. Após 30 dias do procedimento cirúrgico o animal apresentava apoio afetivo dos membros, locomovendo-se normalmente. Mesmo sendo um procedimento desgastante para o ortopedista veterinário, concluímos que é viável e apresenta resultados satisfatórios na realização da ostectomia bilateral concomitante da cabeça do fêmur.
\end{abstract}

Palavras-chave: Artropatia. Cães. Fêmur. Legg-Calve-Perthes. Ortopedia. Radiologia.

\section{BILATERAL ASEPTIC NECROSIS OF THE FEMORAL HEAD IN MONGREL DOG - CASE REPORT}

SUMMARY: The Aseptic Necrosis of the Femoral Head is a crippling disease that causes several limitations in locomotor activities of the animal involved. The highest incidence of early signs of disease is between 6 and 7 months of age, with a range 3-13 months. In this context, this paper aims to report a case of bilateral Aseptic Necrosis of the Femoral Head in a female mongrel dog for about five years of age and weighing $7 \mathrm{~kg}$, with a history of instability and incoordination of hind limbs. As it was young, we opted for the surgical procedure of bilateral ostectomy concomitant femoral head and neck as a treatment for the condition. After 10 days of the procedure, it was observed that the animal had mild support member, the points are removed and found no relevant change in the incision. After 30 days of the surgical procedure the animal had emotional support from members, walking through normally. Even as a stressful procedure for the orthopedic vet concluded that it is feasible and provides satisfactory results the performance of simultaneous bilateral ostectomy of the femoral head.

Keywords: Arthropathy. Dogs. Femur. Legg-Calve-Perthes. Orthopedics. Radiology.

\section{INTRODUÇÃO}

Os distúrbios que acometem as articulações de cães são etiologicamente diversos e podem ser divididos em duas categorias principais: não inflamatórios e inflamatórios; sendo que o principal fator que distingue essas duas classes de doenças articulares é o derramamento de neutrófilos no líquido sinovial nas

\footnotetext{
${ }^{1}$ Prof. Dr. Departamento de Clínica e Cirurgia Veterinária. FAFRAM/FE

${ }^{2}$ Aprimorando em Clínica e Cirurgia Veterinária FAFRAM/FE

${ }^{3}$ Médico(a) Veterinário
} 
doenças inflamatórias. Na classe das não inflamatórias podem ser incluídas as doenças articulares degenerativas, doenças articulares traumáticas, luxações e subluxações, distúrbios dos meniscos, distúrbios dos ossos sesamóides, artropatias neuropáticas, artropatias do desenvolvimento, artropatias secundárias a erros inatos do metabolismo, artropatias dietéticas e neoplasias articulares (PEDERSEN et al., 2004).

A Necrose Asséptica da Cabeça do Fêmur (NACF), também pode ser designada como doença de Legg-Perthes ou Legg-Calvé-Perthes, osteocondrite juvenil, necrose avascular, necrose isquêmica ou coxa plana, é classificada como uma artropatia do desenvolvimento, sendo uma doença incapacitante que provoca diversas limitações nas atividades locomotoras do animal acometido. Definida como sendo autolimitante, caracteriza-se pela morte do tecido ósseo em decorrência da privação de suprimento sanguíneo, parcial ou total, podendo ser de origem traumática, devido a fraturas, luxações e ampla desnudação do periósteo, ou não traumática, em decorrência de longos períodos de corticoterapia, doenças metabólicas, compressão venosa entre outras (CORREIA et al., 1996; DOIGE; WEISBRODE, 1998; SOUZA; SILVA FILHO, 1999; AIELLO, 2001; CARPENTER, 2003; SCHULZ, 2008).

O processo torna a cabeça do fêmur e o quadril deformados, com uma gama limitada de movimento e de riscos futuros para as alterações secundárias artríticas (LAREDO FILHO et al., 2001; SONI et al., 2004; MAMAN et al., 2007).

A NACF geralmente ocorre antes do fechamento fisário da cabeça do fêmur (SCHULZ, 2008). A condição é geralmente unilateral. Porém, em alguns casos podem acometer, de maneira independente, ambas as cabeças femorais em 10 a $17 \%$ dos casos (STURION; STURION, 2000; KEALY; McALLISTER, 2005; FARROW, 2006; SCHULZ, 2008).

A maior incidência de inicio dos sinais da afecção é entre 6 e 7 meses de idade, com uma variação de 3 a 13 meses. Os animais acometidos geralmente apresentam claudicação uni ou bilateral, de inicio lento e piorando num período de 6 a 8 semanas; dor, devido à fragmentação da epífise femoral e a osteoartrite; e, em casos crônicos, pode ocorrer atrofia muscular e impotência funcional do membro afetado, progredindo para a não sustentação do peso pelo animal. Alguns animais podem apresentar, ainda, irritabilidade, inapetência e o ato de morder a pele sobre o membro afetado (GAMBARDELLA, 1996; PIERMATTEI; FLO, 1999; SCHULZ, 2008).

Em 1909, Waldenström descreveu pela primeira vez o fenômeno, porém atribuiu a causa à tuberculose. Em 1910, Arthur Legg nos Estados Unidos, Jacques Calvé na França e George Perthes na Alemanha, descreveram, de maneira independente, essa afecção de etiologia ainda desconhecida em humanos (SONI et al., 2004).

O primeiro relato na literatura em Medicina Veterinária foi feito por Tutt em 1935 (NUNAMAKER, 1985). Desde então, várias pesquisas foram realizadas sobre a afecção, chegando à conclusão de que acomete principalmente cães de pequeno porte e jovens, sendo que machos e fêmeas são igualmente afetados (GAMBARDELLA, 1996; PIEK et al., 1996; OLMSTEAD, 1998; PIERMATTEI; 
FLO, 1999; BIASI et al., 2000; AIELLO, 2001; PEDERSEN et al., 2004; KEALY; McALLISTER, 2005).

Pinscher, Poodles, Yorkshires, Terriers e West Higland White Terriers são as raças que apresentam maior incidência (CARPENTER, 2003; ALLAN, 2007). Ocasionalmente pode ocorrer em cães de raças grandes (OLMSTEAD, 1998). Sendo que gatos, aparentemente, não são afetados pela afecção (SCHULZ, 2008).

Nos animais suspeitos não são observadas alterações laboratoriais consistentes e os sinais clínicos apresentados não demonstram alterações específicas e conclusivas (BIASI et al., 2000; VASSEUR, 2005; SCHULZ, 2008). Assim, o exame radiológico é a técnica indicada, e largamente utilizada, para confirmação diagnóstica (DEMKO; McLAUGHLIN, 2005; FROES, 2011).

Nesse contexto, o presente trabalho objetiva relatar um caso de Necrose Asséptica de Cabeça de Fêmur (NACF) bilateral em um canino sem raça definida (SRD) tratado através da técnica ortopédica de ostectomia da cabeça e colo femorais.

\section{RELATO DO CASO}

Uma cadela recém-adotada, proveniente de rua, sem raça definida (SRD), de aproximadamente cinco anos de idade e pesando $7 \mathrm{~kg}$, foi atendida na Clínica Veterinária Casa dos Bichos, localizada em Cravinhos-SP, apresentando incoordenação motora e instabilidade de membros posteriores. Durante o exame físico foi evidenciado dor a palpação.

No exame radiográfico foi visibilizado diminuição da opacidade óssea da cabeça e colo femorais, perda do contorno arredondado característico, aumento do espaço articular do quadril, acetábulo raso com margem cranial achatada, além de angulação mais aguda entre o colo femoral e a haste femoral (Figura 1).

Figura 1. Imagem fotográfica de exame radiográfico da articulação coxo-femoral de canino SRD apresentando necrose asséptica bilateral de cabeça de fêmur. Observam-se focos de osteólise em ambas as cabeças femorais (círculos). Posição ventro-dorsal. CravinhosSP. 2011.

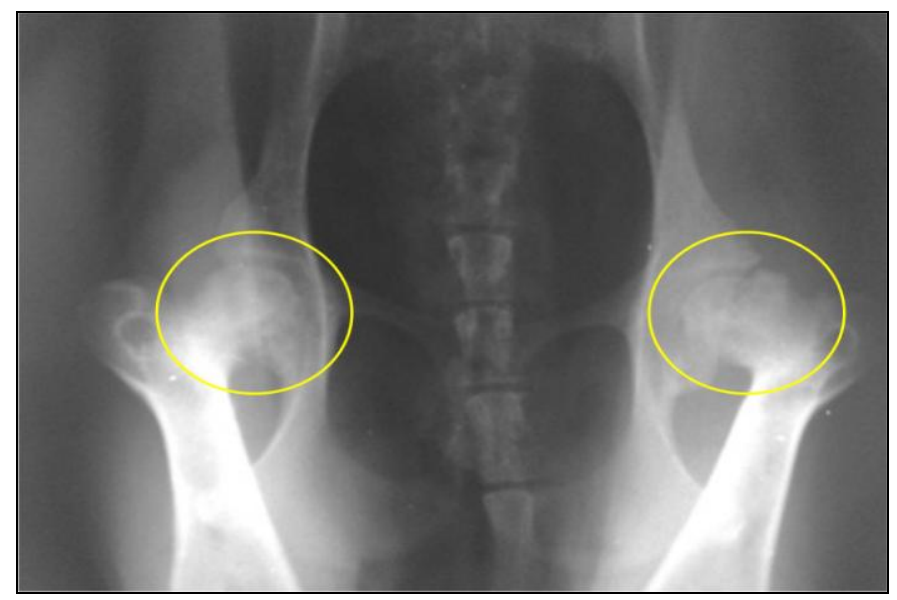


Baseado no histórico, no exame físico e radiográfico realizado, diagnosticou-se a afecção bilateral apresentada pelo animal como Necrose Asséptica da Cabeça do Fêmur (NACF). Como o mesmo era jovem, optou-se pelo procedimento cirúrgico de ostectomia bilateral concomitante de cabeça e colo femorais como tratamento para a afecção.

No intervalo até a realização do procedimento cirúrgico foi prescrito Carprofeno $(2,2 \mathrm{mg} / \mathrm{kg} / \mathrm{VO}$, a cada 12 horas) e recomendado ao proprietário que limitasse o espaço físico de trânsito do animal, suspendesse passeios e demais exercícios que viessem a exigir esforço físico demasiado do mesmo e, que trouxesse o animal sob jejum alimentar e hídrico de oito horas no dia do procedimento cirúrgico.

No dia do procedimento, o proprietário relatou que o animal não respondeu ao tratamento prescrito, persistindo a incoordenação e instabilidade locomotora, além das dores.

O procedimento pré-operatório consistiu em limpeza corporal com água fria, tricotomia e antissepsia prévia da região lombar e dos membros pélvicos, tanto lateral como medial. A veia radial medial foi cateterizada para infusão de solução fisiológica, por onde também foi injetado Fentanil (5,0 $\mathrm{mcg} / \mathrm{kg})$ e Propofol $(5,0 \mathrm{mg} / \mathrm{kg})$ para indução anestésica. Em seguida, o animal foi acomodado em posição esternal, com os membros pélvicos tracionados em sentido cranial, para aplicação epidural de Lidocaína $(4,0 \mathrm{mg} / \mathrm{kg})$. A manutenção anestésica foi feita com Isofluorano por via inalatória.

No centro cirúrgico, o animal foi acomodado em decúbito lateral esquerdo, após antissepsia e isolamento do membro pélvico com o campo estéril, foi iniciado o procedimento cirúrgico. Terminado o procedimento do lado esquerdo, o animal foi acomodado em decúbito lateral direito para exérese da cabeça e colo femoral do mesmo membro.

$\mathrm{O}$ acesso cirúrgico à cabeça e colo femoral, tanto do membro esquerdo como do direito, foi feito conforme técnica proposta por Schulz (2008). Assim sendo, foi feita uma incisão cutânea cranio-lateral centralizada sobre a articulação do quadril. Em seguida, o músculo bíceps femoral foi retraído caudalmente e o músculo tensor da fáscia lata cranialmente. Acessada a cápsula articular, esta foi incisionada. $\mathrm{O}$ membro foi rotacionado externamente, identificada a linha de junção do colo com a metáfise do fêmur e, com auxilio de um osteotomo e de um martelo, procedeu-se a ostectomia da cabeça e colo femorais.

Durante a ostectomia, devido ao alto comprometimento do tecido ósseo, a cabeça femoral do membro direito foi fragmentada em vários pedaços.

Após palpar e conferir a ausência de irregularidades na superfície da incisão, a cápsula articular foi suturada sobre o acetábulo. A musculatura incisada foi suturada com fio Vycril 2.0, a aproximação do tecido subcutâneo foi feita com fio Nylon-2.0 em padrão simples contínuo e a dermorrafia com fio Nylon2.0 em padrão Wolf interrompido. 
Figura 2. Imagem fotográfica de cabeça de fêmur esquerda de canino SRD apresentando necrose asséptica de cabeça de fêmur bilateral após ostectomia cirúrgica. Observa-se perda do contorno arredondado característico. Cravinhos-SP. 2011.

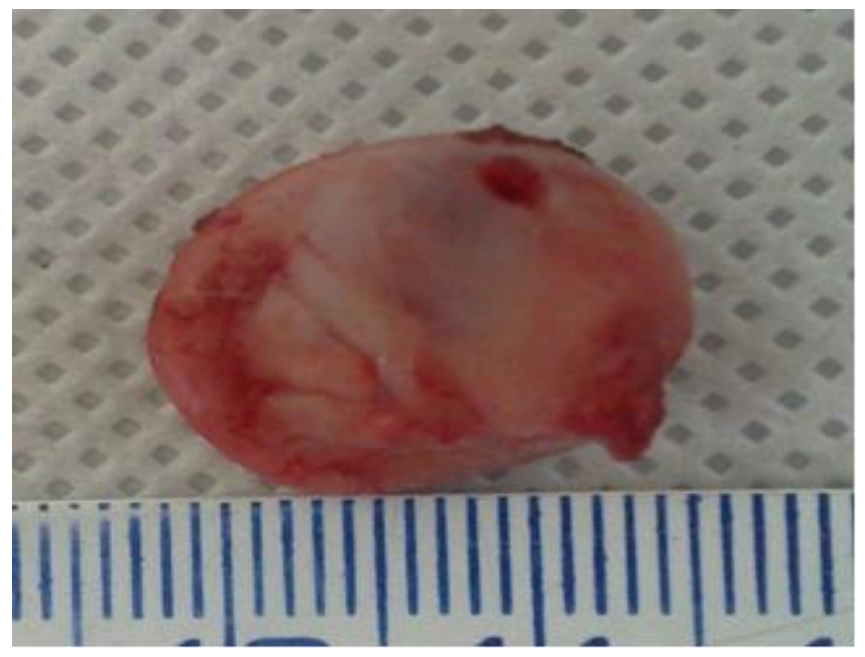

Para o período pós-operatório, foi prescrito limpeza da ferida cirúrgica com solução fisiológica e instilação de Rifampicina (a cada 8 horas, durante 10 dias) e, aplicação de compressa com gelo durante 20 minutos no local (a cada 6 horas, durante 5 dias), além da administração de Dipirona (27,5 mg/kg/VO, a cada 8 horas, durante 10 dias), Cloridrato de Tramadol (2,0 mg/kg/VO, a cada 12 horas, durante 10 dias), Firocoxib (57 mg/VO, a cada 24 horas, durante 30 dias) e antibioticoterapia preventiva baseada na associação de Metronidazol (125 mg/kg/VO, a cada 8 horas, durante 10 dias) e Espiramicina (750.000 UI/VO, a cada 8 horas, durante 10 dias).

As feridas cirúrgicas não apresentaram edema, inflamação, secreção ou rompimentos de pontos, sendo o processo cicatricial considerado satisfatório. Decorridos 10 dias do procedimento, observou-se que o animal apresentava apoio leve dos membros; os pontos foram removidos e não foi encontrada nenhuma alteração relevante no local da incisão. Após 30 dias do procedimento cirúrgico o animal apresentava apoio afetivo dos membros, locomovendo-se normalmente.

\section{DISCUSSÃO}

Como foi sugerido por Olmstead (1998) e Biasi et al. (2000), o diagnóstico de NACF do presente animal foi baseado no histórico de relatado pelo proprietário durante a anamnese, no exame clínico-semiológico assim como, nos achados radiográficos.

Legg, Calvé e Perthes descreveram a entidade clínica, porém somente Legg declarou a mais aceitável patogênese, no qual acreditou ser um defeito no suprimento de sangue da epífise femoral. Calvé acreditava que a condição deveria ser por uma Ricketsia, e Perthes relacionou a doença a uma artrite degenerativa, provavelmente de natureza infecciosa (NUNAMAKER, 1985).

Atualmente, tem-se o conceito de que a NACF é o resultado final de uma combinação de fatores mecânicos e biológicos que levariam a circulação intraóssea da cabeça femoral a um quadro isquêmico 
(PENEDO et al., 1993). Nesse contexto, a maioria dos autores defende que a NACF decorre de uma interrupção do suprimento sanguíneo na porção da epífise femoral relativa à cabeça do fêmur, resultando num colapso e a área afetada sofre necrose. Porém, a etiologia não é totalmente conhecida, mas diversas teorias foram propostas, incluindo a interferência hormonal, fatores hereditários, conformação anatômica, pressão intracapsular e infarto da cabeça do fêmur (BALDERSTON et al, 1996; GAMBARDELLA, 1996; KEALY; McALLISTER, 2005; SCHULZ, 2008).

O suprimento vascular da cabeça do fêmur em animais jovens com as fises femorais proximais abertas é derivado, exclusivamente, dos vasos epifisários, que correm por fora dos ossos, ao longo da superfície do colo femoral, atravessam a placa de crescimento e penetram o osso para nutrir a epífise femoral. A sinovite ou uma posição anormal prolongada do membro pode aumentar a pressão intraarticular, levando ao colapso das veias mais frágeis e à inibição do fluxo sanguíneo (SCHULZ, 2008).

Após a morte celular, o osso responde com um processo reparador. Contudo, o conteúdo ósseo é enfraquecido mecanicamente durante o período de revascularização e as forças de sustentação de peso fisiológicas normais podem causar o colapso e a fragmentação da epífise femoral, ocasionando uma doença articular degenerativa (WISNER; POLLARD, 2007; SCHULZ, 2008) que altera a forma anatômica e favorece a proliferação de osteófitos perivasculares (OLMSTEAD, 1998; PIERMATTEI; FLO, 1999; FROES, 2011).

O perfil do paciente desse relato se enquadra na descrição que refere que o aparecimento dos sintomas clínicos ocorre, na maioria dos animais, entre 5 e 8 meses de idade, podendo variar dos 3 aos 13 meses (PIERMATTEI e FLO 1999; CARPENTER, 2003). SCHULZ (2008) descreve que, durante os estágios iniciais, a NACF não é dolorosa na maioria dos animais, portanto, o diagnóstico é estabelecido, frequentemente, depois que o colapso e a fragmentação tiverem resultado em incongruência e degeneração articular.

O exame radiográfico é a técnica rotineiramente utilizada para estabelecer o diagnóstico, pois possibilita a visibilização e diferenciação de outras afecções devido às características específicas apresentadas pela mesma, assim como, auxilia no planejamento do tratamento e conduta com o paciente (SANTANA FILHO, et al., 2011; FROES, 2011). Segundo Farrow (2006), quando a afecção envolve apenas uma articulação é possível detectar alterações sutis ao comparar-se a articulação afetada com a normal.

$\mathrm{Na}$ imagem radiográfica são visibilizadas frequentemente diminuição da opacidade óssea da cabeça e do colo femorais, perda do contorno arredondado característico e achatamento cranial, aumento do espaço articular do quadril, o acetábulo se torna raso e sua margem cranial apresenta-se achatada, pode ocorrer fragmentação da cabeça do fêmur com descontinuidade do osso subcondral, além de angulação mais aguda entre o colo femoral e a haste femoral e desenvolvimento de uma deformidade vara (OLMSTEAD, 1998; PIERMATTEI; FLO, 1999; KEALY; McALLISTER, 2005). Tais sinais podem ser identificados nas fases iniciais da doença, todavia podem apresentar-se diferentes nas fases mais crônicas. 
Assim como, podem não estar tão evidentes em animais recentemente acometidos pela afecção, recomendando-se repetir o exame num intervalo de dez dias (FROES, 2011).

De acordo com Piermattei; Flo (1999), frequentemente, o primeiro sinal notado é a irritabilidade; o animal pode morder a área do flanco e do osso coxal. A dor pode ser determinada na articulação coxofemoral, particularmente na abdução. Mais tarde, a crepitação pode estar presente com taxa de movimentação restrita e encurtamento do membro. Atrofia dos músculos glúteos e quadríceps tornam-se aparente. A ocorrência de claudicação é geralmente gradual, e seis a oito semanas são necessárias para progressão até a completa impotência funcional. A dor pode ser aguda quando existir fraturas da cabeça femoral em áreas líticas (GAMBARDELLA, 1996; BIASI et al., 2000; AIELLO, 2001; CARPENTER, 2003; PEDERSEN et al., 2004).

Desde o início do tratamento de uma alteração ortopédica deve-se agir de forma a prevenir complicações imediatas e, principalmente, as tardias como pseudoartrose, coxa vara, consolidação viciosa ou fechamento fisário prematuro. Contudo, a NACF é a única, dentre as alterações ortopédicas, que, do momento que iniciou seu curso, não pode ser evitada, mesmo quando é efetuado um atendimento rápido e adequado (ASTUR et al, 2010).

O tratamento da NACF tem sido até agora um problema de difícil solução para os ortopedistas. Fatores como acometimento bilateral, etiologia, idade, localização, estágio e extensão da necrose são variáveis que apresentam combinações múltiplas e, consequentemente, evoluções difíceis de ser previstas, tornando o tratamento dessa doença um verdadeiro desafio (FUJIKI, 1998).

A artroplastia total da articulação coxo-femoral é, atualmente, utilizada em cães com alterações na articulação coxo-femoral, entre elas a NACF, com 92 a 98\% de resultados satisfatórios (DEYOUNG et al., 1992). Porém, segundo Barbosa (2008), para a realidade brasileira, o uso desta técnica ainda é insipiente devido ao seu alto custo, sendo frequente ainda o uso da excisão completa da cabeça e colo femorais.

A excisão da cabeça e colo femoral é considerada o tratamento de escolha em cães (DENNY; BUTTERWORTH, 2000). Optar pelo tratamento cirúrgico, por meio da excisão da cabeça e do colo femorais, é a técnica sugerida por Pedersen et al. (2004) como o tratamento recomendado em casos avançados de NACF. Olmstead (1998) e Piermattei e Flo (1999) afirmam que resultados satisfatórios são obtidos com tal tratamento cirúrgico, além de que o tempo de recuperação é menor e a taxa de sucesso é mais alta do que a do tratamento conservativo.

Pedersen et al. (2004) relatam ainda que, em alguns casos, os animais respondem somente à administração de agentes condroprotetores. O tratamento conservador com anti-inflamatórios e exercícios limitados pela coleira ou sem a sustentação de peso, como natação, pode propiciar o alivio da dor em uma pequena porcentagem dos cães, mas a maioria destes requer intervenção cirúrgica para alivio da claudicação, sendo a excisão da cabeça e do colo do fêmur o tratamento de eleição (SCHULZ, 2008).

Segundo Foganholli; Filadelpho (2006), o uso da acupuntura e as suas técnicas de aplicação têm sido muito bem aceitas por veterinários e proprietários como forma de terapia para diversas afecções, entre 
elas a NACF. Sendo que, dependendo do grau da lesão, se a cura total não puder ser estabelecida, alcançase pelo menos, a melhora na qualidade de vida do paciente.

Segundo Matera et al. (2003), a irradiação periarticular, com o laser de baixa potência Arseneto de Gálio, promove um rápido retorno da função do membro em cães após a excisão artroplástica da cabeça do fêmur, otimizando a recuperação pós-operatória.

Em animais domésticos, devido à convivência pacífica existente com humanos, com rápido acesso ao tratamento e a facilidade de manejo, percebe-se uma boa recuperação dos mesmos (PROBST, 1996; EGGER, 1998; JOHNSON, 2008). Segundo Aiello (2001) e Carpenter (2003), a utilização de sessões de fisioterapia ajuda a estimular o uso do membro afetado. Corroborando com SCHULZ (2008), que descreve que o animal submetido à ostectomia da cabeça do fêmur deve ser estimulado a utilizar o membro imediatamente após a cirurgia, o que deve incluir exercícios de reabilitação física, assim como, quando o animal tolerar, flexão e extensão passivas da articulação do quadril duas vezes ao dia iniciandose com pequenos movimentos e aumentando a amplitude gradativamente.

De acordo com Carpenter (2003), faz-se necessário monitorar os pacientes submetidos ao tratamento cirúrgico, visando avaliar a recuperação cirúrgica, a adaptação biológica e a evolução dos movimentos e comportamento do animal.

O prognóstico quanto à recuperação após a cirurgia é favorável (VASSEUR, 1996; OLMSTEAD, 1998; BIASI et al., 2000; AIELLO, 2001; CARPENTER, 2003; PEDERSEN et al., 2004; BARBOSA, 2008; SCHULZ, 2008). Contudo, ocasionalmente podem ser obtidos resultados insatisfatórios, muitas vezes relacionados à ausência de sustentação de peso antes da cirurgia, a uma atrofia muscular pré-operatória grave ou a realização de uma técnica cirúrgica incorreta (FUJIKI, 1998; SCHULZ, 2008).

Ao deparar-se com casos de NACF bilateral a maioria dos ortopedistas veterinários optam pela ostectomia unilateral intercalada da cabeça do fêmur. Porém, pela nossa experiência clínica, verificamos que alguns proprietários desistem de realizar o segundo procedimento, alegando que o mesmo seria demasiadamente oneroso financeiramente ou, ainda, que o período de recuperação pós-cirúrgico exige muita dedicação ao animal e faltaria tempo pra isso.

\section{CONCLUSÃO}

Mesmo sendo um procedimento desgastante para o ortopedista veterinário, concluímos que é viável e apresenta resultados satisfatórios na realização da ostectomia bilateral concomitante da cabeça do fêmur.

\section{REFERÊNCIAS}

AIELLO, S. E. Manual Merk de veterinária. 8.ed. São Paulo: Roca, 2001. p.495-496, 706. 
ALLAN, G. S. Radiographic signs of joint disease in dogs and cats. In: THRALL, D. E. Textbook of veterinary diagnostic radiology. 5.ed. St. Louis: Saunders Elsevier, 2007. p.317-358.

ASTUR, D. C.; ARLIANI, G. G.; NASCIMENTO, C. L. S.; BLUMETTI, F. C.; FONSECA, M. J. A.; DOBASHI, E. T.; PINTO, J. A.; ISHIDA, A. Correlação entre os índices de necrose e a estabilização precoce nas fraturas da extremidade proximal do fêmur na infância. Revista Brasileira de Ortopedia, v.45, n.4, p.426-432, 2010.

BALDERSTON, R. A.; ROTHMAN, R. H.; BOOTH, R. E.; HOZACK, W. J. O quadril: procedimentos clínicos e cirúrgicos. São Paulo: Revinter, 1996.

BARBOSA, A. L. T. Recuperação funcional coxo-femoral pós-operatória em cães: estudo clínico e biomecânico. 2008. 66f. Dissertação (Mestrado em Cirurgia Veterinária). Centro de Ciências Rurais. Universidade Federal de Santa Maria. Santa Maria-RS.

BIASI, F.; MOTTA, T.; BERGAMO, F. M. M. Afecções ortopédicas de cães e gatos em crescimento. Revista Cães e Gatos, v.95, n.3, p.24-25, 2000.

CARPENTER, L. Necrose asséptica da cabeça do fêmur. In: TLLEY, L. P.; JUNIOR, F. W. K. S. Consulta veterinária em 5 minutos. São Paulo: Manole, 2003, p. 888-889.

CORREIA, M. A.; OSÓRIO, L.; COUTO, P.; CHAMBRIARD, C.; CAIAFFA, V. Avaliação radiológica do componente femoral da prótese bipolar não cimentada no tratamento da necrose avascular da cabeça femoral. Revista Brasileira de Ortopedia, v.31, n.5, p.405-408, 1996.

DEYOUNG, D. J.; DEYOUNG, B. A.; ABERMAN, H. A; KENNA, R. V.; HUNGERFORD, D. S. Implantation of an uncemented total hip prosthesis. Technique and initial results of 100 arthroplasties. Veterinary Surgery, v.21, p.168-177, 1992.

DEMKO, J.; MCLAUGHLIN, R. Developmental orthopedic disease. Veterinary Clinics of North America: Small Animal Practice, v.35, n.5, p.1111-1135, 2005.

DOIGE, C. E.; WEISBRODE, S. E. Doenças dos ossos e das articulações. In: CARLTON, W. W.; McGAVIN, M. D. Patologia especial de Thomson. 2.ed. Porto alegre, 1998. p.448-485.

DENNY, H. R.; BUTTERWORTH, S. J. A guide to canine and feline orthopedic surgery. 4.ed. Oxford: Blackwell Science, 2000. 496 p.

EGGER, E. L. Fraturas do rádio e ulna. In: SLATTER, D. Manual de cirurgia de pequenos animais. São Paulo: Manole, 1998. p.2057-2079.

FARROW, C. S. Infecção, neoplasia e outras doenças da pelve. In: imagem do cão e do gato. São Paulo: Roca, 2006. p.330-333. Veterinária: diagnóstico por

FOGANHOLLI, J. N.; FILADELPHO, A. L. Tratamento de distúrbios neuromusculares em cães com o uso da acupuntura. Revista Científica Eletrônica de Medicina Veterinária, v.3, n.7, 2006. Disponível em: http://www.revista.inf.br/veterinaria07/revisao/Edic08-revisao01.pdf. Acesso: 25 nov. 2011.

FROES, T. R. Diagnóstico radiográfico das doenças articulares pélvicas do cão. Veterinária e Zootecnia em Minas, v.21, n.110, p.35-39, 2011.

FUJIKI, E. N. Tratamento da necrose avascular da cabeça do fêmur não traumática, grau III de Ficat, pela osteotomia transtrocantérica de rotação, técnica de Sugioka. Revista Brasileira de Ortopedia, v.33, n.10, p.753-758, 1998. 
GAMBARDELA, P. C. Moléstia de Legg-Calva-Perthes em cães. In: BOJRAB, M. J. Mecanismo da moléstia na cirurgia de pequenos animais. Barueri-SP: Manole, 1996. p.933-937.

JOHNSON, A. L. Fundamentos de cirurgia ortopédica e manejo de fraturas. In: FOSSUM, T. W. et al. Cirurgia de pequenos animais. 3.ed. São Paulo: Roca, 2008. p.930-1014.

KEALY, J. K.; MCALLISTER, H. Radiologia e ultra-sonografia do cão e do gato. 3.ed. Barueri-SP: Manole, 2005. 436p.

LAREDO FILHO, J.; DIAS, L. S.; KUWAJIMA, S. S. Doença de Legg-Calvé-Perthes. Clínica Ortopédica, v.2, p.71-90, 2001.

MAMAN, E.; YANIV, M.; SEGEV, E. Legg-Calve-Perthes disease: literature review and updates.

Harefuah, v.146, n.1, p.68-72, 2007.

MATERA, J. M.; TATARUNAS, A. C.; OLIVEIRA, S. M. Uso do laser arsenato de gálio (904nm) após excisão artroplástica da cabeça do fêmur em cães. Acta Cirúrgica Brasileira, v.18, n.2, p.102-106, 2003.

NUNAMAKER, D. M. Legg-Calvé-Perthes. In: NEWTON, C. D.; NUNAMAKER, D. M. Textbook of small animal orthopedics. Philadelphia: JB Lippincott; 1985. p. 949-952.

OLMSTEAD, M. L. Articulação coxofemoral. ln: BIRCHARD, S. I.; SHERDING, R. G. Manual Saunders: Clínica de pequenos animais. São Paulo: Roca, 1998, p. 1138-1139.

PEDERSEN, N. C.; MORGAN, J. P.; VASSEUR, P. B. Doenças articulares de cães e gatos. In: ETTINGER, S. J.; FELDMAN, E. C. Tratado de medicina interna veterinária: doenças do cão e do gato. Rio de Janeiro: Guanabara Koogan, 2004. p.1971-1988.

PENEDO, J. L.; RONDINELLI, P.; CABRAL, F. P.; FREITAS, E. Necrose avascular da cabeça femoral: tratamento pela técnica de descompressão cirúrgica. Revista Brasileira de Ortopedia, v.28, n.6, p.338$342,1993$.

PIEK, C. J.; HAZEWINKEL, H. A. W.; WOLVEKAMP, W. T. C.; NAP, R. C.; MEY, B. P. Long-term follow-up of avascular necrosis of the femoral head in the dog. Journal of Small Animal Practice, v.37, n.1, p.12-18, 1996.

PIERMATTEI, D. L.; FLO, G. L. Manual de ortopedia e tratamento das fraturas dos pequenos animais. São Paulo: Manole, 1999. p. 433-434.

PROBST, C. W. Membro torácico. In: BOJRAB, M. J. Técnicas atuais em cirurgia de pequenos animais. 2.ed. São Paulo: Roca, 1996. p.692-757.

SANTANA FILHO, M. V.; ATAN, J. B. C. D.; MUlleR, L. C. C. M.; ESPOSITO, C. C.; SILVA, P. C.; FERREIRA, M. L. Doença de Legg-Calvé-Perthes: revisão bibliográfica. Pubvet, v.5, n.9. Disponível em: http://www.pubvet.com.br/imagens/artigos/342011-170418-ferreira1052.pdf. Acesso: 05 nov. 2011.

SCHULZ, K. Afecções articulares. In: FOSSUM, T. W. et al. Cirurgia de pequenos animais. 3.ed. Rio de Janeiro: Elsevier, 2008. p.1143-1315.

SONI, J.; VALENZA, W.; SCHELLE, G. Doença de Legg-Calvé-Perthes. In: Ortopedia pediátrica. Rio de Janeiro: Revinter, 2004. p.185-194.

SOUZA, I.; SILVA FILHO, N. M. Necrose avascular da cabeça femoral induzida por corticóide: estudo experimental. Revista Brasileira de Ortopedia, v.34, n.7, p.439-446, 1999. 
STURION, D. J.; STURION, M. A. T. Radiologia veterinária. Londrina: Edunopar, 2000. 287p.

VASSEUR, P. B. Ostectomia da cabeça e do colo femorais. In: BOJRAB, M. J. Técnicas atuais em cirurgia de pequenos animais. 3.ed. São Paulo: Roca, 2005. p.634-642.

WISNER, E. R.; POLLARD, R. E. Orthopedic diseases of young and growing dogs and cats. In:

THRALL, D. E. Textbook of veterinary diagnostic radiology. 5.ed. St. Louis: Saunders Elsevier, 2007. p.268-283. 
